\title{
Axonal transport proteins as biomarkers of neurodegeneration?
}

\author{
Kelly Hares ${ }^{1}$ \& Alastair Wilkins* ${ }^{*} 1$ \\ ${ }^{1}$ Institute of Clinical Neurosciences, University of Bristol, Southmead Hospital, Bristol, Bristol, UK BS 10 5NB \\ * Author for correspondence: Tel.: +44 0117 4146695; alastair.wilkins@bristol.ac.uk
}

First draft submitted: 9 May 2017; Accepted for publication: 17 May 2017; Published online: 3 August 2017

Keywords: Alzheimer's disease $\bullet$ axonal transport $\bullet$ biomarkers $\bullet$ kinesin $\bullet$ multiple sclerosis $\bullet$ neurodegeneration

Neurodegeneration is a cardinal feature of numerous neurological diseases, yet the mechanisms underlying neurodegeneration are disparate and are not currently fully understood. In addition, the diagnosis of neurodegenerative conditions is often challenging and treatments are limited. Nevertheless, new therapeutic strategies and trials are being developed and, as a result, better biomarkers of disease severity, progression and prognosis are required in order to monitor the efficacy of new treatments.

\section{Axonal transport}

Since studies have shown that dysregulated axonal transport may precede neurodegeneration, understanding the relationship between axonal transport and neurodegeneration is of interest [1]. The constant transport of proteins from the cell body, through the axon toward the synapse, is imperative to optimal signal conductance and neuronal function. Transport of proteins through the axon is mainly mediated by two families of motor proteins, kinesin and dynein. Kinesins govern the majority of anterograde transport and dynein is responsible for retrograde transport, shuttling proteins back toward the cell body for recycling. Dynein motors are relatively few in number and indirectly bind to proteins through dynactin. In contrast, there are over 40 different members of the kinesin superfamily motor protein (KIF) family with various splice variants, highlighting the complexity of anterograde axonal transport and protein localization [2].

The original 'conventional' kinesin (kinesin I/KIF5) was shown to be a tetrameric protein composed of two heavy chains $(110-120 \mathrm{kDa})$ and two light chains $(60-70 \mathrm{kDa})$. Further studies have shown that kinesin heavy chain consists of three basic domains: a head, stalk and tail domain. The head $\left(\mathrm{NH}_{2}\right.$-terminal) usually contains a microtubule-binding domain and a conserved globular motor domain which hydrolyses ATP to produce the energy required for movement of cargoes along the microtubule [3]. The tail domain (COOH-terminal) is where membranous organelles (cargoes) bind. Cargoes often bind indirectly to the C-terminal through a receptor-adaptor complex or through association with kinesin light chains (KLCs; as for the conventional kinesin structure [KIF5]). Protein cargo specificity is determined by the KIF carboxy tail domain [2]. Cargoes transported by conventional kinesin include neurofilaments (NFs), mitochondria, amyloid precursor protein (APP) and SNARE complex proteins [4].

\section{Axonal transport disruption \& neurodegeneration}

NFs play an integral role in axonal structure. Local phosphorylation of NFs within the axon increases their chargedbased negative repulsion which in turn, optimizes axonal caliber for signal conductance [5]. Both dephosphorylation and hyperphosphorylation of NFs have been implicated in various neurodegenerative conditions including, multiple sclerosis (MS), Alzheimer's disease (AD), Parkinson's disease and amyotrophic lateral sclerosis [6,7]. Indeed, histopathologically, large aggregates of proteins such as NFs, APP and $\tau$ are found in both the cell body and axon in various neurological conditions, including neurofibrillary tangles and $\mathrm{A} \beta$ plaques in $\mathrm{AD}$; axonal spheroids in MS and Lewy bodies in Parkinson's disease. Large protein aggregates suggest disruption of axonal transport mechanisms and may be associated with subsequent neurodegeneration. In support of this theory, studies using familial AD APP-overexpressing transgenic mice have shown abnormal axonal morphology and large axonal swellings co-positive 
for KLC and phosphorylated NF (heavy chain) within 4 months of birth [8]. In addition, transgenic mice with modified PSEN1 expression have demonstrated reduced affinity between KLC and membrane-bound organelles (such as synaptophysin and syntaxin-I-containing vesicles), mediated via KLC phosphorylation by elevated glycogen synthase kinase $3 \beta$ activity [9]. Other studies in mice have shown that knock-out of conventional kinesin member KIF5A is neonatal lethal, and postnatal targeting of the gene results in reduced axon caliber, eventual axon loss and hindlimb paralysis [10]. Interestingly, mutations in the KIF5A gene have been linked to various axonopathies including hereditary spastic paraplegia type 10 and Charcot-Marie-Tooth disease type 2A [11,12]. Spastic paraplegia type 10 is implicated in disturbed intracellular axonal transport and is characterized by axonal loss in the corticospinal tract. More recently, single nucleotide polymorphisms (SNPs) within the KIF5A gene locus have been linked to MS susceptibility [13]. Additionally, KIF5A is reduced in MS post-mortem brain tissue compared to control, and differences in KIF5A protein expression in MS patients based on the presence or absence of susceptibility SNPs, suggesting that they may play a role in disease progression [14]. There is also a significant inverse correlation between KIF5A expression and that of its associated cargoes (phosphorylated NF and APP) in MS white matter, suggesting protein aggregates commonly found in MS are influenced by deficiencies in anterograde transport, potentially through reduced motor protein KIF5A expression. KIF5A is believed to transport several cargoes through association with KLCs, including APP, phosphorylated NFs, presynaptic membrane proteins SNAP-25 and syntaxin-1b. Some studies have linked SNPs in the KLC1 gene to AD and suggested that genetic variability in the KLC1 gene may influence the development of $\mathrm{AD}$ [15]. This SNP has been shown to predict conversion of mild cognitive impairment to $\mathrm{AD}$ and correlates with hyperphosphorylated $\tau$ levels in cerebrospinal fluid (CSF). It is hypothesized that the polymorphism could affect cargo-binding affinity by structural changes in the C-terminal. Reduced or dysregulated transport could cause structural instability to the neuron, which may result in neuronal degeneration. In mice, deletion of the KLC1 subunit leads to early selective defects of axonal transport of several cargoes including APP, NF and $\tau$ aggregates, causing cytoskeletal disorganization and neurodegeneration [16]. One study in human tissue has shown reduced KLC1 protein expression in AD frontal cortex [17]. Understanding how build-up of these substances occurs and therapies to reduce aggregate formation are key areas of drug development.

\section{Using axonal transport proteins as biomarkers of neurodegeneration?}

One of the major unmet needs in neurodegeneration research is the availability of biomarkers: to provide more accurate diagnosis, prognosis and outcome measures in clinical trials. Developing biomarkers which reflect early tissue injury and which are linked to early onset of disability would inform on treatment decisions and as a result, may reduce the long-term burden of disease within individuals. The complex pathophysiology of neurodegenerative conditions makes development of biomarkers challenging since analysis of one marker alone may not be able to detect significant correlation between its levels and clinical disease outcome measures. In neurodegenerative diseases, tissue damage causes the release of various intracellular proteins into the CSF, which can be measured as potential disease biomarkers. CSF biomarkers as potential prognostic indicators have been studied for some time. Axonal injury and loss are closely linked to disease progression in MS, as documented in several radiological and postmortem human tissue studies [18]. Concerning axonal protein biomarkers, the main NF subunits: light, medium and heavy have been found to be elevated in MS CSF compared with controls [19], however, confounding data exist in terms of their individual effectiveness at determining disease progression. In $\mathrm{AD}$, the most commonly measured CSF proteins are $\mathrm{A} \beta$ and $\tau$, with some studies showing differences between frontotemporal lobar dementia and $A D$. Interestingly, APOE $\varepsilon 4$ genotype has been shown to correlate with higher levels of CSF A $\beta$, which draws attention to the potential behind genotyping for biomarker studies [20]. A major advance in understanding the pathology of neurodegenerative disease has come about in recent years by results from large genome-wide association studies. These have revealed a large number of susceptibility genes and suggested a variety of putative therapeutic targets for drug development. Genetic factors are important not only for susceptibility to disease but also, in all likelihood, for disease course. With this in mind, future studies should seek to develop a novel biomarker of axonal injury which links genetic factors to markers of tissue injury.

The current evidence suggests axonal transport proteins may be worthy candidates for biomarkers of neurodegeneration. It may be that certain KIF genotypes are more linked to certain types or degrees of neuronal or axonal injury which may help to explain the wide variability in neurodegenerative phenotypes. At present, KIFs have not been used as a CSF biomarker, but it is possible to detect their presence in CSF and, as stated, dysregulated axonal transport may precede neurodegeneration, so their presence may be an early indicator of disease before irreversible damage has set in [1]. Determining whether specific genetic variations in axonal motor proteins (which can be tested 
in the clinical setting) influence disease progression, would undoubtedly have huge implications in the development of personalized treatments for various neurodegenerative conditions, allowing clinicians more informed choices when deciding therapies as well as enabling patients to have a greater understanding of how their disease is likely to progress in the future.

\section{Financial \& competing interests disclosure}

The authors have no relevant affiliations or financial involvement with any organization or entity with a financial interest in or financial conflict with the subject matter or materials discussed in the manuscript. This includes employment, consultancies, honoraria, stock ownership or options, expert testimony, grants or patents received or pending, or royalties.

No writing assistance was utilized in the production of this manuscript.

\section{References}

1 Morfini GA, Burns M, Binder LI et al. Axonal transport defects in neurodegenerative diseases. J. Neurosci. 29(41), 12776-12786 (2009).

2 Hirokawa N, Noda Y. Intracellular transport and kinesin superfamily proteins, KIFs: structure, function, and dynamics. Physiol. Rev. 88(3), 1089-1118 (2008).

3 Hirokawa N. Kinesin and dynein superfamily proteins and the mechanism of organelle transport. Science 279(5350), 519-526 (1998).

4 Hirokawa N, Noda Y, Tanaka Y, Niwa S. Kinesin superfamily motor proteins and intracellular transport. Nat. Rev. Mol. Cell Biol. 10(10), 682-696 (2009).

5 Petzold A, Gveric D, Groves $\mathrm{M}$ et al. Phosphorylation and compactness of neurofilaments in multiple sclerosis: indicators of axonal pathology. Exp. Neurol. 213(2), 326-335 (2008).

6 Julien JP, Mushynski WE. Neurofilaments in health and disease. Prog. Nucleic Acid Res. Mol. Biol. 61, 1-23 (1998).

7 Trapp BD, Peterson J, Ransohoff RM, Rudick R, Mork S, Bo L. Axonal transection in the lesions of multiple sclerosis. N. Engl. J. Med. 338(5), 278-285 (1998).

8 Stokin GB, Lillo C, Falzone TL et al. Axonopathy and transport deficits early in the pathogenesis of Alzheimer's disease. Science 307(5713), 1282-1288 (2005).

9 Pigino G, Pelsman A, Mori H, Busciglio J. Presenilin-1 mutations reduce cytoskeletal association, deregulate neurite growth, and potentiate neuronal dystrophy and tau phosphorylation. J. Neurosci. 21(3), 834-842 (2001).

10 Xia CH, Roberts EA, Her LS et al. Abnormal neurofilament transport caused by targeted disruption of neuronal kinesin heavy chain KIF5A. J. Cell Biol. 161(1), 55-66 (2003).

11 Reid E, Kloos M, Ashley-Koch A et al. A kinesin heavy chain (KIF5A) mutation in hereditary spastic paraplegia (SPG10). Am. J. Hum. Genet. 71(5), 1189-1194 (2002).

12 Crimella C, Baschirotto C, Arnoldi A et al. Mutations in the motor and stalk domains of KIF5A in spastic paraplegia type 10 and in axonal Charcot-Marie-Tooth type 2. Clin. Genet. 82(2), 157-164 (2012).

13 Alcina A, Fedetz M, Fernandez O et al. Identification of a functional variant in the KIF5A-CYP27B1-METTL1-FAM119B locus associated with multiple sclerosis. J. Med. Genet. 50(1), 25-33 (2013).

14 Hares K, Redondo J, Kemp K, Rice C, Scolding N, Wilkins A. Axonal motor protein KIF5A and associated cargo deficits in multiple sclerosis lesional and normal-appearing white matter. Neuropathol. Appl. Neurobiol. 43(3), 227-241 (2016).

15 Andersson ME, Sjolander A, Andreasen N et al. Kinesin gene variability may affect tau phosphorylation in early Alzheimer's disease. Int. J. Mol. Med. 20(2), 233-239 (2007).

16 Falzone TL, Stokin GB, Lillo C et al. Axonal stress kinase activation and tau misbehavior induced by kinesin-1 transport defects. J. Neurosci. 29(18), 5758-5767 (2009).

17 Morel M, Heraud C, Nicaise C, Suain V, Brion JP. Levels of kinesin light chain and dynein intermediate chain are reduced in the frontal cortex in Alzheimer's disease: implications for axoplasmic transport. Acta Neuropathol. 123(1), 71-84 (2012).

18 Bjartmar C, Kidd G, Mork S, Rudick R, Trapp BD. Neurological disability correlates with spinal cord axonal loss and reduced N-acetyl aspartate in chronic multiple sclerosis patients. Ann. Neurol. 48(6), 893-901 (2000).

19 Fitzner B, Hecker M, Zettl UK. Molecular biomarkers in cerebrospinal fluid of multiple sclerosis patients. Autoimmun. Rev. 14(10), 903-913 (2015).

20 Menendez-Gonzalez M. Biomarkers in neurodegenerative disorders: translating research into clinical practice. Front. Aging Neurosci. 6, 281 (2014). 